\title{
Update on Dementia with Lewy Bodies
}

\section{Stella Karantzoulis • James E. Galvin}

Published online: 21 June 2013

(C) Springer Science+Business Media New York 2013 disease (AD), with prevalence rates of up to $5 \%$ in the elderly population and up to $30 \%$ of all dementia cases [1, $2 \bullet \bullet$. Despite considerable research and now better-known diagnostic criteria for DLB, large autopsy series continue to highlight low diagnostic sensitivity for the clinical syndrome of DLB in dementia patients, even among expert clinicians in specialized centers [3-5]. Early and more accurate detection of DLB is therefore needed for optimal management of this complex dementia syndrome, to offer patients and caregivers the appropriate information about the expected symptomatology and disease course, allow for effective pharmacotherapy to be started, and avoid the adverse consequences of neuroleptic sensitivity.

\section{Clinical Diagnosis}

Consensus criteria were developed by the Consortium on DLB International Workshop for the Clinical Diagnosis of DLB, last revised in $2005[2 \bullet \cdot$. Like all dementias, there is a progressive reduction in cognitive functioning that interferes with daily functioning. The rate at which the cognitive impairment progress in DLB is generally similar to $[6 \bullet, 7]$ or shows a steeper rate of decline [5] relative to AD. A study by Stavitsky and colleagues [8] is one exception to this, in which $\mathrm{AD}$ patients had a steeper rate of decline on cognitive and behavioral measures, although the patients with DLB were more impaired at baseline. Reports have shown that the mean survival time in DLB is comparable to [5] or shorter [9] than $\mathrm{AD}$.

Core features for the clinical diagnosis include fluctuating cognition, recurrent well-formed visual hallucinations, and spontaneous parkinsonism. Extrapyramidal signs, including bradykinesia, facial masking, and rigidity usually have a bilateral presentation and can vary in severity. Resting tremor is less common and axial rigidity is more common in DLB compared with Parkinson's disease (PD).

Additional clinical features suggestive of DLB include rapid eye movement sleep behavior disorder (RBD) and severe neuroleptic sensitivity with increased parkinsonism, and a higher prevalence of neuroleptic malignant syndrome. 
A suggestive imaging feature for DLB is low dopamine transporter uptake in the basal ganglia demonstrated by SPECT or PET imaging. Often present but not specific features of DLB include repeated falls and syncope, transient and unexplained loss of consciousness, autonomic dysfunction, hallucinations in other modalities, systematized delusions, depression, relative preservation of medial temporal lobe structures on structural neuroimaging, reduced occipital activity on functional neuroimaging, prominent slow wave activity on electroencephalogram (EEG), and low uptake iodine-123 metaiodobenzylguanidine (MIBG) myocardial scintigraphy. Two core features (or one core feature and one suggestive feature) are sufficient for a diagnosis of probable DLB; one core feature, or one or more suggestive features, is suggestive for a diagnosis of possible DLB [2••]. As with AD, definitive diagnosis of DLB rests on brain autopsy.

Although these features may support the clinical diagnosis of DLB, they lack diagnostic sensitivity and can be seen in other neurodegenerative disorders. In general, these criteria permit high specificity ( $95 \%$ ), but lower sensitivity (83\%), for the presence of neocortical LBs [10]. The accuracy of these criteria is also dependent on the populations assessed, with greater predictive accuracy of cases with relatively rare 'pure' forms of DLB than the more common cases that have a mixture of DLB and AD pathology (sensitivity is approximately $12 \%$ in mixed DLB/AD cases; [4]).

\section{Distinguishing DLB from AD}

For patients with DLB, it is almost impossible to determine the extent to which Alzheimer pathology contributes to their profile of cognitive impairment [11]. Many patients with $A D$ can also show mild parkinsonism and psychiatric symptoms, which further complicates clinical differentiation of DLB from $\mathrm{AD}$. These clinical features in the $\mathrm{AD}$ patient may be attributable to tau pathology rather than LB pathology. Visual hallucinations and visuospatial/constructional dysfunction may be the best predictors for DLB [12], and individuals with visual hallucinations performed significantly worse than those without hallucinations on tasks of angle, objectform, and space-motion discrimination [13]. A recent report based on caregiver responses [14] indicated that the most common presenting symptom in DLB was memory impairment $(57 \%)$, followed by visual hallucinations (44\%), depression (34\%), problem-solving difficulties (33\%), gait problems (28\%), and tremor/stiffness (25\%). Only $3 \%$ reported visual hallucinations as a presenting symptom in $\mathrm{AD}$, with almost ( $99 \%$ ) reporting memory impairment as a presenting symptom in AD. The presence of visual hallucinations together with memory impairment may therefore be the best predictor of DLB and its differentiation from AD.

Patients with DLB generally have early, prominent deficits on tests of executive functions (e.g., judgment, organization, planning) relative to patients with $\mathrm{AD}[15,16]$, although, this is not always the case [17]. Recent work suggests that frontal subcortical circuits associated with executive dysfunction, disinhibition, and apathy are actually affected early in $\mathrm{AD}$ but not until later stages in DLB [17]. Simple attention can be normal in both DLB and AD (as assessed by digit span tasks); however, group differences (DLB $>A D$ ) more often emerge on complex attentional tasks, such as those requiring mental control, visual search and set shifting, and visual selective attention [18]. Patients with pure LB pathology typically have better verbal memory skills than those with pure $\mathrm{AD}$ or mixed DLB/AD [6•]. Patients with pure AD and mixed DLB/AD show equivalent degrees of impairment on verbal memory testing. In contrast, combined AD and LB pathology appears to have an additive effect on visual memory skills.

A milder naming deficit is noted in DLB than in AD, as assessed by measures of confrontation naming, although the naming deficit in DLB may become progressively more severe with disease progression [15]. On measures of generative fluency, DLB patients tend to be equally impaired in category and letter fluency, whereas AD patients show disproportionate difficulty on tests of category relative to letter fluency, present early on in the disease course [19]. This may be related to differences in underlying mechanisms: whereas AD patients have degraded semantic networks or retrieval difficulties affecting semantic networks, attentional and executive impairments likely contribute to the difficulties with word search and retrieval in DLB.

\section{Distinguishing DLB from PDD}

A related disorder that shares many of the same clinical and cognitive features as DLB is Parkinson's disease dementia (PDD) [20]. Pure DLB appears to have a clinical picture more similar to PDD [20] than to AD, with initially more severe executive dysfunction compared with memory deficits [21]. In the majority of patients, however, DLB and PDD are easy to differentiate clinically by the predominance of dementia in DLB $[2 \cdot \bullet]$ and extrapyramidal motor features in patients with PD [22]; nevertheless, in some patients, dementia and extrapyramidal signs occur in close succession, provoking debate about their nosology. For research purposes, cases are classified as DLB if dementia occurs within 12 months of the parkinsonian features, but as PDD if dementia occurs a year or more after a clinical diagnosis of PD $[2 \bullet \bullet, 20]$. In real clinical practice, a diagnosis is made based on the prominent clinical features. The separation between DLB and PDD is considered by some to be artificial, since such a separation implies that the two clinical syndromes have different anatomical substrates, which may ultimately have different therapeutic challenges, and there is little difference in the distribution or severity of LB pathology between PDD and DLB [23]. 


\section{Behavioral and Neuropsychiatric Features}

In DLB, visual hallucinations tend to be well-formed, detailed, most commonly involving people (often described as dysmorphic or small), animals, body parts, and machines. Auditory hallucinations can occur in a subset of people with visual hallucinations. Visual hallucinations are typically present early in the course of the disease and do not resolve with disease progression. In an analysis of autopsy-confirmed cases, hallucinations and delusions were more frequent with LB pathology (75\%) than $\mathrm{AD}(21 \%)$ at the time of the initial clinical evaluation [24]. This was also true for those cases with mixed DLB and AD (53\%) pathology relative to $\mathrm{AD}$ alone. The occurrence of visual hallucinations in the first 4 years after dementia onset has a positive and negative predictive value for DLB of $81 \%$ and $79 \%$, respectively [25]. Visual hallucinations are linked to cholinergic depletion in the temporal cortex and the basal forebrain [26], and may predict a good response to cholinesterase inhibitors [27]. Delusions occur in more than half (56\%) of DLB patients at first presentation and in just over two-thirds $(65 \%)$ at some point in their illness. Delusions tend to be more common in DLB than in PDD or AD. Paranoid, Caprgras (believing individuals are replaced by identical imposter) and "phantom boarder" (unseen individuals residing in one's home) are among the most common content of the delusions. Misidentification syndromes are particularly prevalent in DLB occurring in up to $40 \%$ of patients, compared to $10 \%$ in $\mathrm{AD}$ [28].

Depression is common in DLB and there is equivocal evidence as to whether base rates of depressed mood and major depression differs between DLB and AD [25]. A history of depression has been reported in $58 \%$ of persons with PDD, $50 \%$ of patients with DLB, and in $14 \%$ of AD cases coming to autopsy [29]. Apathy is also common in DLB, particularly with more severe dementia.

\section{Personality Changes}

Personality changes in DLB tend to occur with auditory and visual hallucinations and include diminished emotional responsiveness, resignation of hobbies, increased apathy, and purposeless hyperactivity [30]. Galvin and colleagues [30] reported patients with DLB were more likely to manifest personality traits associated with passive personality traits (e.g., diminished emotional responsiveness, relinquished hobbies, growing apathy, and purposeless activity) than patients with AD.

\section{Cognitive Fluctuations}

Fluctuations in cognition are one of the hallmarks of DLB, seen in $15-80 \%$ of DLB cases [31]. They often involve waxing and waning of cognition, attention, concentration, functional abilities, or arousal in the absence of any clear precipitant. They can be described as episodes of behavioral confusion, inattention, hypersomnolence, and incoherent speech alternating with episodes of lucidity and capable task performance. Patients may be described as staring into space or dazed and the episodes can last minutes to days, varying from alertness to stupor.

A number of scales have been developed to assess cognitive fluctuations, including the Clinical Assessment of Fluctuation and the One Day Fluctuation Assessment Scale, and the Mayo Fluctuations Questionnaires [25]. The Mayo scale describes four features of fluctuations that can reliably distinguish DLB from AD or normal aging including: (1) being drowsy or lethargic during the day despite sleeping the night before; (2) sleeping for more than 2 hours during the day before $7 \mathrm{pm}$; (3) have periods of disorganized or illogical thoughts; and (4) staring spells. Three or four features of this composite occurred in $63 \%$ of patients who had DLB compared with $12 \%$ of those who had $\mathrm{AD}$ and $0.5 \%$ of normally functioning elderly. The presence of three or four of these features yielded a positive predictive value of $83 \%$ for the clinical diagnosis of DLB versus AD.

\section{Autonomic Dysfunction}

Autonomic features are prominent in many cases of DLB and are often reported by the patient and family as among the most disturbing features of the disease. Constipation is a frequent early complaint and may precede any cognitive or motor symptoms by more than a decade. Symptomatic orthostasis is probably the most serious manifestation of autonomic dysfunction, observed in approximately $15 \%$ of DLB patients, and can markedly increase the risk of falls. Other features include decreased sweating, sialorrhea, seborrhea, heat intolerance, urinary dysfunction, diarrhea, and erectile dysfunction/impotence. DLB patients also have a higher frequency of carotid hypersensitivity than elderly patients or patients with AD. There is also evidence of cardiac denervation by iodine-123 metaiodobenzylguanidine (MIBG) scanning, a finding not seen in AD.

\section{Sleep Disorders}

Parasomnias are common in DLB. REM sleep behavior disorder (RBD) is the most common parasomnia and it tends to begin concurrently or after the onset of parkinsonism or dementia, but can be seen up to 20 years before the onset of cognitive or movement symptoms [32]. RBD is marked by lack of normal muscle atonia that prevents movements (other than eye movements) during REM sleep in the presence of excessive activity while dreaming; this can result in vocalizations 
and sometimes wildly violent behavior. RBD is more commonly found in men in their $50 \mathrm{~s}$, and may precede the clinical signs of DLB by many years.

\section{Neuroleptic Sensitivity}

DLB patients are notorious for their neuroleptic sensitivity. Reactions are observed in $30-50 \%$ of DLB patients, characterized by sudden onset of impaired cognition, acute confusion, psychotic episodes, and exacerbation of parkinsonism symptoms such as rigidity and immobility [33]. In some cases, these reactions including neuroleptic malignant syndrome can lead to death within several days. One survey [34] revealed a $58 \%$ frequency of neuroleptic sensitivity to olanzapine, with lower rates with clozapine $(11 \%)$ and thioridazine $(6 \%)$. These data support the notion of unacceptable safety profiles for some neuroleptics in DLB. DLB patients also tend to be activated by sedatives and awakened by sleep medications [35].

\section{Improving Clinical Recognition: Using Risk Scores}

Application of clinical criteria improves specificity of the diagnosis of probable DLB but lacks sensitivity in general practice. One of the great challenges in differential diagnosis of neurodegenerative disorders is attributing clinical symptoms to specific pathologies to guide treatment choices and discuss prognosis and clinical course. We previously used autopsy-confirmed cases to examine clinical features that predict the presence of Lewy body pathology [36]. Features that predicted Lewy bodies include extrapyramidal signs, cognitive fluctuations, hallucinations and sleep disturbances. We used this information to develop a Lewy Body Composite Risk Score (Table 1) as a clinical tool that may increase the likelihood of determining whether Lewy Bodies are contributing pathology to the cognitive diagnosis. We applied the Lewy Body Risk Composite Score to a sample of 69 well-characterized individuals ( 25 healthy controls, $24 \mathrm{AD}$ and $20 \mathrm{DLB}$ ) with detailed clinical and cognitive assessments (Table 2). There were no differences in age or education between groups. Global dementia ratings with the Clinical Dementia Rating or the Mini Mental State Exam revealed no differences between $\mathrm{AD}$ and DLB. We also applied z-scores for cognitive performance for global cognitive performance, working memory and visuospatial abilities derived from previous factor analyses [37]. DLB and AD performed similarly in Global and Working memory domains but DLB was significantly more impaired that AD in visuospatial performance. The Lewy Body Composite Risk Scores were significantly different in DLB patients compared with Controls and $\mathrm{AD}$ (both $p<0.001$ ). Receiver operator characteristic curves demonstrated that the Lewy Body Composite Risk Score discriminated individuals likely to have Lewy body pathology from those who likely do not (area under the curve $0.968 ; 95 \%$ CI: 0.93-1.0). Using a cutoff of 3, the Lewy Body Composite Risk Score provide a sensitivity of $90 \%$ and a specificity of $87 \%$.

\section{Genetics}

A family history of dementia may be more common in DLB relative to controls [38] suggesting in some cases an autosomal dominant inheritance [39]. Variants in all three members of the synuclein gene family, $\alpha$ - synuclein, $\beta$ - synuclein, and $\gamma$-synuclein, may affect the risk of developing DLB [40••]. $\gamma$-synuclein is the most recent synuclein member to be linked to LB neuropathology and the least well understood. $\gamma$-synuclein (persyn) is largely expressed in the cell bodies and axons of primary sensory neurons, sympathetic neurons, and motor neurons as well as in brain [41]. $\beta$-synuclein is highly localized to presynaptic sites in neocortex, hippocampus, and thalamus [42] and it may act as a biological negative regulator of $\alpha$-synuclein. It is associated with DLB and not PD; two novel $\beta$-synuclein point mutations, $\mathrm{P} 123 \mathrm{H}$ and $\mathrm{V} 70 \mathrm{M}$, were found in highly conserved regions of the $\beta$-synuclein gene in respective familial $(\mathrm{P} 123 \mathrm{H})$ and sporadic (V70M) DLB index cases [43]. Genetic mutations in the $\alpha$-synuclein gene are most commonly associated with PD, with or without dementia. However, an E46K mutation in the $\alpha$-synuclein gene has been found in one Spanish family with DLB and triplication of the $\alpha$-synuclein gene occurs in a Swedish American family with early-onset parkinsonism and dementia.

Common genetic factors for the susceptibility of DLB have been identified in the glucocerebrosidase (GBA) gene $[44,45 \cdot$. A significantly higher heterozygote frequency for two common GBA gene mutations (N370S and L444P) occurs in patients with PD (2.9\%) or DLB (3.5\%) compared with controls $(0.4 \%)$ [46].

A novel locus for DLB has been identified in Belgian families [47]. A genome-wide scan and further fine-mapping of candidate loci revealed the locus linkage to 2q35-q36 adjacent to the previously reported PARK11 locus. However, screening of five candidate genes has not yet revealed the disease-causing mutation [48]. Further analysis of AD and PD causative genes in patients with DLB has been performed, showing copy number variants in $A P P, S N C A$, and PARK2 genes [44].

\section{Neuroimaging}

Select neuroimaging techniques support a clinical diagnosis of DLB (versus AD). Generalized atrophy, white matter 
Table 1 Lewy Body Composite Risk Score

Please rate the following symptoms as being present or Yes No absent for at least three times over the past 6 months. Does the patient...

Have slowness in initiating and maintaining movement or have frequent hesitations or pauses during movement?

Have rigidity (with or without cogwheeling) on passive range of motion in any of the four extremities?

Have a loss of postural stability with or without frequent falls?

Have a tremor at rest in any of the four extremities or head?

Have excessive daytime sleepiness and/or seem drowsy and lethargic when awake?

Have episodes of illogical thinking or incoherent, random thoughts?

Have frequent staring spells or periods of blank looks?

Have attention, alertness or concentration problems that wax and wane (i.e. fluctuate) over the course of day?

Appear to act out his/her dreams (kick, punch, thrash, shout or scream) while still asleep?

Have visual hallucinations (see things not really there)?

TOTAL SCORE

(C)2013 Lewy Body Composite Risk Score, James E. Galvin and New York University

changes, and rates of progression of atrophy are not helpful in differential diagnosis of DLB from other types of dementia. Volumetric magnetic resonance imaging (MRI) in patients with comparable severity of dementia, on the other hand, suggests that relative preservation of the medial temporal lobe is observed in DLB compared to AD; this can be used to facilitate differential diagnosis between the two disorders [48].

Occipital hypoperfusion on SPECT [49•] or hypometabolism on PET [50] is more typical of DLB than AD, and represents a potential marker of DLB. Occipital hypometabolism and relative preservation of the posterior cingulate gyrus distinguished DLB from AD patients with a sensitivity of $77 \%$ and a specificity of $80 \%$ [51]. Greater metabolic decrease in the anterior cingulate has also been shown in DLB than PDD [52].

Nigrostriatal degeneration, with profound reductions in the striatal dopamine transporter, has consistently been found in DLB patients at autopsy. Nigrostriatal integrity is assessed in vivo with specific ligands, such as ${ }^{123}$ I-FP-CIT in SPECT imaging, which binds to dopamine transporter and $\left[{ }^{11} \mathrm{C}\right]$ dihydrotetrabenazine in PET imaging, which binds to the vesicular monamine transporter. Reduced striatal dopamine transporter uptake in DLB but not in AD has been shown in SPECT studies, which may facilitate in differential diagnosis [53, 54]. A large multicenter study examining ${ }^{123}$ IFP-CIT SPECT in DLB reported a sensitivity of $78 \%$ for detecting probable DLB and a specificity of $90 \%$ for excluding non-DLB, primarily AD [55]. In another study, ${ }^{123} \mathrm{I}$ FP-CIT imaging was in line with postmortem diagnoses in 19 of 20 cases, and was more precise than clinical diagnosis [54]. However, ${ }^{123}$ I-FP-CIT is limited in that it does not discriminate DLB from other atypical parkinsonian syndromes. Nevertheless, head to head analyses comparing ${ }^{123}$ I-FP-CIT imaging to other imaging modalities including $\left(\left[{ }^{18} \mathrm{~F}\right]\right.$ FDG PET have shown superior diagnostic accuracy with ${ }^{123}$ I-FP-CIT imaging [51, 56].

Reduction of striatal dopamine uptake was observed in patients with DLB using $\left[{ }^{18} \mathrm{~F}\right]$ fluorodopa, with a sensitivity of $86 \%$ and a specificity of $100 \%$, although this study was limited by a small sample size [57]. These findings were confirmed in a later study using $\left[{ }^{11} \mathrm{C}\right]$ dihydrotetrabenazine, which also showed significantly reduced uptake in caudate nucleus and anterior and posterior putamen in DLB and PD compared to $\mathrm{AD}$ and normal controls [58]. The International Consensus Criteria for DLB $[2 \cdot \bullet]$ and the European Federation of Neurological Societies (EFNS) guidelines both support the use of dopaminergic SPECT to differentiate between DLB and AD [59]. A limited number of PET studies have examined the amyloid burden in DLB and PDD in vivo [60, 61], suggesting that cortical amyloid burden was higher in DLB than in PDD, but similar to that in AD.

\section{Therapeutic Approaches}

There are no approved therapies specifically for DLB; however there are a number of reports in the literature regarding
Table 2 Sample characteristics for validation of Lewy Body Composite Risk Score

Mean (SD)

$A D$ Alzheimer disease; $D L B$ dementia with Lewy bodies; $C D R$ $S B$ Clinical Dementia Rating Sum of Boxes; MMSE Mini Mental Status Exam; $L B C R$ Lewy Body Composite Risk; $n s$ not significant

\begin{tabular}{lllll}
\hline & Controls $(N=25)$ & AD $(N=24)$ & DLB $(N=20)$ & $p$ value \\
\hline Age, y & $72.6(6.4)$ & $74.8(6.8)$ & $72.6(8.4)$ & $\mathrm{ns}$ \\
Gender, \% male & 28.0 & 58.3 & 70.0 & 0.01 \\
Education, y & $15.2(1.9)$ & $13.7(2.9)$ & $15.4(2.7)$ & $\mathrm{ns}$ \\
CDR-SB & $0.02(0.11)$ & $3.4(1.8)$ & $3.9(2.9)$ & $<0.001$ \\
MMSE & $28.9(1.2)$ & $25.4(3.2)$ & $25.3(3.5)$ & $<0.001$ \\
Global, z-score & $0.19(0.65)$ & $-0.85(0.78)$ & $-0.86(0.77)$ & $<0.001$ \\
Working, z-score & $0.13(0.81)$ & $-0.57(0.76)$ & $-0.56(0.67)$ & $<0.001$ \\
Visuospatial, z-score & $0.25(0.70)$ & $-0.56(1.0)$ & $-1.65(1.5)$ & $<0.001$ \\
LBCR Score & $0.8(1.4)$ & $1.0(1.1)$ & $5.8(2.2)$ & $<0.001$ \\
\hline
\end{tabular}


the use of medications approved for other disorders that may be considered for symptomatic treatment of DLB.

\section{Cognitive Symptoms}

It has been suggested that treatment with acetylcholinesterase inhibitors (AChEIs) may be more effective in DLB than $\mathrm{AD}$, due to early and prominent central nervous system cholingergic dysfunction in this group [62]. In one study, patients with DLB showed greater treatment improvement in cognition with AChEIs relative to $\mathrm{AD}$ patients, although the difference between the two groups was not significant [63]. Only a few clinical trials for the use of AChEIs in DLB have been conducted, and most guidelines are based on case reports and extension of therapeutic trials in AD. Three independent clinical studies of AChEIs treatment using donepezil, galantamine, and rivastigmine in patients with DLB suggest that acetylcholinesterase inhibitors improve cognitive and neuropsychiatric measures, with no significant increase in EPS with AChEIs use. Currently, there is no compelling evidence that any one AChEIs is better than the other. A recent Cochrane review suggest the effect of ACHEIs in DLB is unclear [64]. Results have been variable in a few case reports or case series in patient with DLB using the NMDA antagonist memantine, another treatment modality approved for use in $\mathrm{AD}$; in these trials, both worsening [65] and improvement [66] were noted.

\section{Motor Symptoms}

There are no controlled clinical trials that have yet evaluated the treatment of motor features in DLB. There are reports of small series of DLB patients whose motor impairments were successfully treated with levodopa [67]. Levodopa is more effective in treating motor impairments in idiopathic PD than DLB. Dopamine agonists are associated with more side effects, especially drug-induced psychosis, even at low doses. Given this risk and because the motor impairments may be mild, the recommendation is to treat the movement disorder only if the motor symptoms interfere with function. Antiparkinsonism medicines should be initiated at the lowest possible dose and increased with caution. There have been reports of increased adverse events with the combined use of levodopa and cholinesterase inhibitors in PD [68]. Cholinesterase inhibitors can potentially exacerbate parkinsonism. In a study of rivastigmine in PD, approximately $10 \%$ of patients treated with rivastigmine had worsening of tremor [69]. However, their overall motor function was not significantly different between the treatment and placebo groups [69]. Other PD medications such as amantadine, catechol-O-methyltransferase inhibitors, monoamine oxidase inhibitors, and anticholinergics tend to exacerbate cognitive impairment and should ideally be avoided.

\section{Behavioral Symptoms}

There are no approved medications for behavioral treatment in DLB. Clinical experience suggests that nonpharmacologic treatment approaches should be considered first, including evaluating for physical ailments that may be provoking behavioral disturbances (e.g., fecal impaction, pain or decubitus ulcers). Avoidance or reduction of doses of other medications that can potentially cause agitation should also be attempted. Generally speaking, when medications are needed to modify behaviors, they should be used at the lowest dose and for the shortest duration possible.

Visual hallucinations have been considered predictors of a good response to AChEIs [70]. Classical neuroleptics (such as haloperidol) are best avoided in DLB as they may worsen motor function and even potentially result in life-threatening neuroleptic sensitivity. Experience with atypical antipsychotics in DLB has been mixed. Risperidone and olanzapine have been shown to control psychosis and agitation in $\mathrm{AD}$ in randomized trials. Although low doses of risperidone $(0.5 \mathrm{mg})$ and olanzapine $(2.5 \mathrm{mg})$ are usually well tolerated and do not usually result in motor deterioration [71], motor deterioration may still be seen in advanced DLB [72].

Randomized, placebo-controlled studies showing significant improvement of psychosis without worsening of motor symptoms have only been shown with clozapine, although this was not specifically in individuals with DLB [73]. However, given the potentially fatal adverse event of agranulocytosis and need for blood monitoring, clozapine is generally not a first line treatment. Quetiapine has become a popular treatment of psychosis given the low incidence of motor deterioration and its ability to control visual hallucinations at low doses. Efficacy and tolerability has been documented in both PD and DLB [73]. REM sleep behavior disorder generally responds to low doses of clonazepam prior to sleep.

\section{Autonomic Dysfunction}

Management of orthostatic hypotension includes relatively simple measures such as leg elevation, elastic stockings, increasing salt and fluid intake, and if not medically contraindicated, avoiding medications that can exacerbate orthostasis. Medications such as midodrine or fludrocortisone can be used in the event that such simple measures fail. Midodrine is a vascoconstrictor and side effects include urine retention and supine 
hypertension. Fludrocortisone has mineralcorticoid activity and causes fluid retention.

Medications with anticholinergic activity, including oxybutynin, tolterodine tartrate, berthanechol chloride, and propantheline can be used to treat urinary urgency, frequency, and urge incontinence. As they can potentially exacerbate cognitive problems, these medications should be used cautiously. As DLB is more common in males, the risk of producing urine retention in the setting of prostatic hypertrophy should be considered.

\section{Conclusions}

This review has summarized the major advances that have taken place in recent years in understanding the mechanisms underlying DLB, its clinical diagnosis, and its management. It is a complicated, common dementia syndrome affecting the elderly, characterized by varying degrees of cognitive, behavioral, affective, movement, and autonomic dysfunction. Despite more well-known criteria for this dementia syndrome, last revised in 2005, cases continue to be missed most often are misdiagnosed as AD. DLB and PDD differ chiefly in the relative timing of dementia and parkinsonism. Early amyloid deposits in DLB relative to PDD may account for their difference in the timing of dementia and parkinsonism.

As with the other neurodegenerative dementias, earliest possible diagnosis of DLB, even prior to symptom onset, is central to developing effective therapeutic strategies, but this relies upon the development of robust, specific, and sensitive biologic or cognitive markers as diagnostic tools and therapeutic endpoints. We provide evidence that using a composite risk score increases the likelihood that Lewy bodies are present and contributing to the clinical syndrome. This would be true for both DLB and PDD. Recently identified genetic mutations, candidate markers from biological fluids, and new imaging modalities may improve understanding of disease mechanisms. Still, challenges remain in defining their correlation with pathological processes and their ability to detect DLB and related disorders. It may be that a combined approach is needed to distinguish DLB from its look-alikes.

Acknowledgements This effort was supported by grants from the National Institutes of Health, P30 AG008051 and R01 AG040211, and the Michael J Fox Foundation. Dr. Galvin and New York University are the copyright holders for the Lewy Body Composite Risk Score.

\section{Compliance with Ethics Guidelines}

Conflict of Interest Stella Karantzoulis and James E. Galvin declare that they have no conflict of interest.
Human and Animal Rights and Informed Consent This article does not contain any studies with human or animal subjects performed by any of the authors.

\section{References}

Papers of particular interest, published recently, have been highlighted as:

- Of importance

•. Of major importance

1. Zaccai JC, McCracken C, Brayne C. A systematic review of prevalence and incidence studies of dementia with Lewy bodies. Age Ageing. 2005;34(6):561-6.

2. $\cdot$ McKeith IG et al. Diagnosis and management of dementia with Lewy bodies: third report of the DLB Consortium. Neurology. 2005;65(12):1863-72. This study outlines the latest revision of the consensus criteria on $D L B$.

3. Shim YS, et al. Clinicopathologic study of Alzheimer's disease: Alzheimer Mimics. J Alzheimers Dis. 2013 March 12 [Epub ahead of print].

4. Nelson PT et al. Low sensitivity in clinical diagnoses of dementia with Lewy bodies. J Neurol. 2010;257(3):359-66.

5. Nelson PT et al. Relative preservation of MMSE scores in autopsyproven dementia with Lewy bodies. Neurology. 2009;73(14):1127-33.

6. - Johnson DK, Morris JC, Galvin JE. Verbal and visuospatial deficits in dementia with Lewy bodies. Neurology. 2005;65(8):1232-8. This study examined the cognitive decline in $D L B$ and showed that comorbid presence of $D L B$ and $A D$ alters the cognitive presentation of visuospatial deficits in dementia, but does not alter dementia progression.

7. Hanyu $\mathrm{H}$ et al. Differences in clinical course between dementia with Lewy bodies and Alzheimer's disease. Eur J Neurol. 2009;16(2):212-7.

8. Stavitsky K et al. The progression of cognition, psychiatric symptoms, and functional abilities in dementia with Lewy bodies and Alzheimer disease. Arch Neurol. 2006;63(10):1450-6.

9. Williams MM et al. Survival and mortality differences between dementia with Lewy bodies vs Alzheimer disease. Neurology. 2006;67(11):1935-41.

10. McKeith IG. Spectrum of Parkinson's disease, Parkinson's dementia, and Lewy body dementia. Neurol Clin. 2000;18(4):865-902.

11. Merdes AR et al. Influence of Alzheimer pathology on clinical diagnostic accuracy in dementia with Lewy bodies. Neurology. 2003;60(10):1586-90.

12. Tiraboschi $\mathrm{P}$ et al. What best differentiates Lewy body from Alzheimer's disease in early-stage dementia? Brain. 2006;129(Pt 3):729-35.

13. Mosimann UP et al. Visual perception in Parkinson disease dementia and dementia with Lewy bodies. Neurology. 2004;63(11):2091-6.

14. Auning $\mathrm{E}$ et al. Early and presenting symptoms of dementia with lewy bodies. Dement Geriatr Cogn Disord. 2011;32(3):202-8.

15. Collerton D et al. Systematic review and meta-analysis show that dementia with Lewy bodies is a visual-perceptual and attentionalexecutive dementia. Dement Geriatr Cogn Disord. 2003;16(4):229-37.

16. Simard M, van Reekum R, Cohen T. A review of the cognitive and behavioral symptoms in dementia with Lewy bodies. J Neuropsychiatry Clin Neurosci. 2000;12(4):425-50.

17. Peavy GM et al. Neuropsychiatric features of frontal lobe dysfunction in autopsy-confirmed patients with lewy bodies and "pure" Alzheimer disease. Am J Geriatr Psychiatry. 2013;21(6):509-19.

18. Hansen L et al. The Lewy body variant of Alzheimer's disease: a clinical and pathologic entity. Neurology. 1990;40(1):1-8. 
19. Lambon R et al. Semantic memory is impaired in both dementia with Lewy bodies and dementia of Alzheimer's type: a comparative neuropsychological study and literature review. J Neurol Neurosurg Psychiatry. 2001;70(2):149-56.

20. Emre $\mathrm{M}$ et al. Clinical diagnostic criteria for dementia associated with Parkinson's disease. Mov Disord. 2007;22(12):1689-707.

21. Kraybill ML et al. Cognitive differences in dementia patients with autopsy-verified AD, Lewy body pathology, or both. Neurology. 2005;64(12):2069-73.

22. Johnson DK, Galvin JE. Longitudinal changes in cognition in Parkinson's disease with and without dementia. Dement Geriatr Cogn Disord. 2011;31(2):98-108.

23. Aarsland D, Ballard CG, Halliday G. Are Parkinson's disease with dementia and dementia with Lewy bodies the same entity? J Geriatr Psychiatry Neurol. 2004;17(3):137-45.

24. Weiner MF et al. Can alzheimer's disease and dementias with Lewy bodies be distinguished clinically? J Geriatr Psychiatry Neurol. 2003;16(4):245-50.

25. Ferman TJ et al. Neuropsychological differentiation of dementia with Lewy bodies from normal aging and Alzheimer's disease. Clin Neuropsychol. 2006;20(4):623-36.

26. Harding AJ, Broe GA, Halliday GM. Visual hallucinations in Lewy body disease relate to Lewy bodies in the temporal lobe. Brain. 2002;125(Pt 2):391-403.

27. McKeith I et al. Dementia with Lewy bodies. Lancet Neurol. 2004;3(1):19-28.

28. Ballard $\mathrm{C}$ et al. Psychiatric morbidity in dementia with Lewy bodies: a prospective clinical and neuropathological comparative study with Alzheimer's disease. Am J Psychiatry. 1999;156(7):1039-45.

29. Klatka LA, Louis ED, Schiffer RB. Psychiatric features in diffuse Lewy body disease: a clinicopathologic study using Alzheimer's disease and Parkinson's disease comparison groups. Neurology. 1996;47(5):1148-52.

30. Galvin JE et al. Personality traits distinguishing dementia with Lewy bodies from Alzheimer disease. Neurology. 2007;68(22):1895-901.

31. Ballard CG et al. Fluctuations in attention: PD dementia vs DLB with parkinsonism. Neurology. 2002;59(11):1714-20.

32. Boeve BF et al. Pathophysiology of REM sleep behaviour disorder and relevance to neurodegenerative disease. Brain. 2007;130(Pt 11):2770-88.

33. McKeith I et al. Neuroleptic sensitivity in patients with senile dementia of Lewy body type. BMJ. 1992;305(6855):673-8.

34. Aarsland D et al. Neuroleptic sensitivity in Parkinson's disease and parkinsonian dementias. J Clin Psychiatry. 2005;66(5):633-7.

35. Rogan S, Lippa CF. Alzheimer's disease and other dementias: a review. Am J Alzheimers Dis Other Demen. 2002;17(1):11-7.

36. Galvin JE et al. Clinical phenotype of Parkinson's disease dementia. Neurology. 2006;67(9):1605-11.

37. Johnson $\mathrm{K}$ et al. Cognitive profils in dementia: Alzheimer disease versus nondemented aging. Neurology. 2008;71(22):1783-9.

38. Woodruff BK et al. Family history of dementia is a risk factor for Lewy body disease. Neurology. 2006;66(12):1949-50.

39. Harding AJ et al. Identification of families with cortical Lewy body disease. Am J Med Genet B Neuropsychiatr Genet. 2004;128B(1):11822.

40. •- Nishioka $\mathrm{K}$ et al. Association of alpha-, beta-, and gammaSynuclein with diffuse lewy body disease. Arch Neurol. 2010;67(8):970-5. This study showed that variants in all three members of the synuclein gene family affect the risk of developing $D L B D$ and warrant further investigation in larger, pathologically defined data sets.

41. Buchman VL et al. Persyn, a member of the synuclein family, influences neurofilament network integrity. Nat Neurosci. 1998;1(2):101-3.

42. Iwai A et al. The precursor protein of non-A beta component of Alzheimer's disease amyloid is a presynaptic protein of the central nervous system. Neuron. 1995;14(2):467-75.
43. Ohtake $\mathrm{H}$ et al. Beta-synuclein gene alterations in dementia with Lewy bodies. Neurology. 2004;63(5):805-11.

44. Meeus B et al. DLB and PDD: a role for mutations in dementia and Parkinson disease genes? Neurobiol Aging. 2012;33(3):629 e5-18.

45. - Nalls MA, Duran R, Lopez G, et al. A multicenter study of glucocerebrosidase mutations in dementia with lewy bodies. JAMA Neurol. 2013;15:1-9. doi:10.1001/jamaneurol.2013.1925. [Epub ahead of print] This study is the largest to date linking mutations in Glucocerebrosidase to DLB.

46. Mata IF et al. Glucocerebrosidase gene mutations: a risk factor for Lewy body disorders. Arch Neurol. 2008;65(3):379-82.

47. Bogaerts V et al. A novel locus for dementia with Lewy bodies: a clinically and genetically heterogeneous disorder. Brain. 2007;130(Pt 9):2277-91.

48. Whitwell JL et al. Focal atrophy in dementia with Lewy bodies on MRI: a distinct pattern from Alzheimer's disease. Brain. 2007;130(Pt 3):708-19.

49. - Colloby SJ et al. Multivariate spatial covariance analysis of 99mTc-exametazime SPECT images in dementia with Lewy bodies and Alzheimer's disease: utility in differential diagnosis. J Cereb Blood Flow Metab. 2013;33(4):612-8. This study showed that multivariate analysis of blood flow SPECT data is robust and shows good diagnostic accuracy in two independent cohorts for distinguishing $D L B$ from $A D$.

50. Minoshima $\mathrm{S}$ et al. Alzheimer's disease versus dementia with Lewy bodies: cerebral metabolic distinction with autopsy confirmation. Ann Neurol. 2001;50(3):358-65.

51. Lim SM et al. The 18F-FDG PET cingulate island sign and comparison to 123I-beta-CIT SPECT for diagnosis of dementia with Lewy bodies. J Nucl Med. 2009;50(10):1638-45.

52. Yong SW et al. A comparison of cerebral glucose metabolism in Parkinson's disease, Parkinson's disease dementia and dementia with Lewy bodies. Eur J Neurol. 2007;14(12):1357-62.

53. O'Brien JT et al. Dopamine transporter loss visualized with FP-CIT SPECT in the differential diagnosis of dementia with Lewy bodies. Arch Neurol. 2004;61(6):919-25.

54. Walker $Z$ et al. Differentiation of dementia with Lewy bodies from Alzheimer's disease using a dopaminergic presynaptic ligand. J Neurol Neurosurg Psychiatry. 2002;73(2):134-40.

55. McKeith I et al. Sensitivity and specificity of dopamine transporter imaging with 123I-FP-CIT SPECT in dementia with Lewy bodies: a phase III, multicentre study. Lancet Neurol. 2007;6(4):305-13.

56. Colloby SJ et al. A comparison of $99 \mathrm{mTc}$-exametazime and 123IFP-CIT SPECT imaging in the differential diagnosis of Alzheimer's disease and dementia with Lewy bodies. Int Psychogeriatr. 2008;20(6):1124-40.

57. Hu XS et al. 18F-fluorodopa PET study of striatal dopamine uptake in the diagnosis of dementia with Lewy bodies. Neurology. 2000;55(10):1575-7.

58. Koeppe RA et al. Differentiating Alzheimer's disease from dementia with Lewy bodies and Parkinson's disease with $(+)-[11 \mathrm{C}]$ dihydrotetrabenazine positron emission tomography. Alzheimers Dement. 2008;4(1 Suppl 1):S67-76.

59. Hort $\mathrm{J}$ et al. EFNS guidelines for the diagnosis and management of Alzheimer's disease. Eur J Neurol. 2010;17(10):1236-48.

60. Edison P et al. Amyloid load in Parkinson's disease dementia and Lewy body dementia measured with [11C]PIB positron emission tomography. J Neurol Neurosurg Psychiatry. 2008;79(12):1331-8.

61. Gomperts SN et al. Imaging amyloid deposition in Lewy body diseases. Neurology. 2008;71(12):903-10.

62. Touchon $\mathrm{J}$ et al. Response to rivastigmine or donepezil in Alzheimer's patients with symptoms suggestive of concomitant Lewy body pathology. Curr Med Res Opin. 2006;22(1):49-59.

63. Samuel W et al. Better cognitive and psychopathologic response to donepezil in patients prospectively diagnosed as dementia with Lewy bodies: a preliminary study. Int $\mathrm{J}$ Geriatr Psychiatry. 2000;15(9):794-802. 
64. Rolinski M, Fox C, Maidment I, McShane R. Cholinesterase inhibitors for dementia with Lewy bodies. Parkinson's disease dementia and cognitive impairment in Parkinson's disease. Cochrane Database Syst Rev. 2012;3, CD006504. doi:10.1002/ 14651858.CD006504.pub2.

65. Ridha BH, Josephs KA, Rossor MN. Delusions and hallucinations in dementia with Lewy bodies: worsening with memantine. Neurology. 2005;65(3):481-2.

66. Sabbagh MN et al. The use of memantine in dementia with Lewy bodies. J Alzheimers Dis. 2005;7(4):285-9.

67. Molloy $\mathrm{S}$ et al. The role of levodopa in the management of dementia with Lewy bodies. J Neurol Neurosurg Psychiatry. 2005;76(9):1200-3.

68. Okereke CS et al. Concurrent administration of donepezil $\mathrm{HCl}$ and levodopa/carbidopa in patients with Parkinson's disease: assessment of pharmacokinetic changes and safety following multiple oral doses. Br J Clin Pharmacol. 2004;58 Suppl 1:41-9.

69. McKeith I et al. Efficacy of rivastigmine in dementia with Lewy bodies: a randomised, double-blind, placebo-controlled international study. Lancet. 2000;356(9247):2031-6.

70. McKeith IG, Mosimann UP. Dementia with Lewy bodies and Parkinson's disease. Parkinsonism Relat Disord. 2004;10 Suppl 1:S15-8.

71. Katz IR et al. Comparison of risperidone and placebo for psychosis and behavioral disturbances associated with dementia: a randomized, double-blind trial. Risperidone Study Group. J Clin Psychiatry. 1999;60(2):107-15.

72. Walker $Z$ et al. Olanzapine in dementia with Lewy bodies: a clinical study. Int J Geriatr Psychiatry. 1999;14(6):459-66.

73. Friedman JH, Fernandez HH. Atypical antipsychotics in Parkinsonsensitive populations. J Geriatr Psychiatry Neurol. 2002;15(3):156-70. 